Revista Arbitrada Interdisciplinaria KOINONIA

Año 2020. Vol V. N³. Especial: Administración

Hecho el depósito de Ley: FA2016000010

ISSN: 2542-3088

FUNDACIÓN KOINONIA (F.K). Santa Ana de Coro. Venezuela.

Segundo Noé Quizhpilema-Acero; Ana Gabriela Hinojosa-Caballero; Renán Teodoro Rodríguez-Pillaga; Juan Edmundo Álvarez-Gavilanes

http://dx.doi.org/10.35381/r.k.v5i3.899

\title{
Diagnóstico de emprendimientos de producción de fresa en la ciudad de Cañar
}

Diagnosis of strawberry production enterprises in the city of Cañar

\author{
Segundo Noé Quizhpilema-Acero \\ segundo.quizhpilema@psg.ucacue.edu.ec \\ Universidad Católica de Cuenca, Cuenca \\ Ecuador \\ https://orcid.org/0000-0001-6253-0646 \\ Ana Gabriela Hinojosa-Caballero \\ aghinojosac@ucacue.edu.ec \\ Universidad Católica de Cuenca, Cuenca \\ Ecuador \\ https://orcid.org/0000-0001-6885-0384 \\ Renán Teodoro Rodríguez-Pillaga \\ rtrodriguezp@ucacue.edu.ec \\ Universidad Católica de Cuenca, Cuenca \\ Ecuador \\ https://orcid.org/0000-0002-5456-5619 \\ Juan Edmundo Álvarez-Gavilanes \\ juan.alvarezg@ucacue.edu.ec \\ Universidad Católica de Cuenca, Cuenca \\ Ecuador \\ https://orcid.org/0000-0003-0978-3235
}

Recibido: 20 de mayo de 2020

Revisado: 15 de junio de 2020

Aprobado: 30 de julio de 2020

Publicado: 15 de agosto de 2020 
Segundo Noé Quizhpilema-Acero; Ana Gabriela Hinojosa-Caballero; Renán Teodoro Rodríguez-Pillaga; Juan Edmundo Álvarez-Gavilanes

\title{
RESUMEN
}

El objetivo consistió en desarrollar un diagnóstico en los emprendimientos de la producción de la fresa del cantón Cañar con el objetivo de identificar los principales problemas y así elevar las utilidades. Se fundamentó centralmente en el paradigma postivista de observación, medición y verificación, con un enfoque cuantitativo y un tipo descriptivo de diseño de campo no experimental. Una de las deficiencias más sobresalientes que se puede resaltar es que los emprendedores se dedican a comercializar el producto solo en el área local, lo cual conlleva a que tengan una visualización muy reducida de la comercialización, además impide que no se tenga una perspectiva para la innovación limitando los ingresos. El modelo de diagnóstico que se utiliza permite efectuar comparaciones entre distintas áreas de la organización para de esta forma identificar el área con mayores problemas y corregirlos en primeras instancias.

Descriptores: Empresa privada; economía de mercado; oferta y demanda; comercio. (Palabras tomadas del Tesauro UNESCO).

\begin{abstract}
The objective consisted of developing a diagnosis in the strawberry production undertakings of the Cañar canton in order to identify the main problems and thus increase profits. It was based centrally on the postivist paradigm of observation, measurement and verification, with a quantitative approach and a descriptive type of non-experimental field design. One of the most outstanding deficiencies that can be highlighted is that entrepreneurs are dedicated to marketing the product only in the local area, which leads to a very reduced visualization of the marketing, and also prevents not having a perspective for the innovation limiting revenue. The diagnostic model used allows comparisons between different areas of the organization to identify the area with the greatest problems and correct them in the first instance.
\end{abstract}

Descriptors: Private enterprises; market economy; supply and demand; trade. (Words taken from the UNESCO Thesaurus). 
Revista Arbitrada Interdisciplinaria KOINONIA

Año 2020. Vol V. №3. Especial: Administración

Hecho el depósito de Ley: FA2016000010

ISSN: 2542-3088

FUNDACIÓN KOINONIA (F.K). Santa Ana de Coro. Venezuela.

Segundo Noé Quizhpilema-Acero; Ana Gabriela Hinojosa-Caballero; Renán Teodoro Rodríguez-Pillaga; Juan Edmundo Álvarez-Gavilanes

\section{INTRODUCCIÓN}

En el Ecuador desde siempre han existido serios problemas en la economía y en el empleo, por tal razón toda la sociedad en los últimos años ha visto la necesidad de emprender y crear su propio empleo. Desde entonces surgen series de micro-empresas en todos los ámbitos a nivel de todo el país elevando la economía y mejorando la calidad de vida de la sociedad. En el 2017 Ecuador es uno de los países más Emprendedores de Latinoamérica junto con Perú, Chile y Colombia. Con la Actividad Emprendedora Temprana (TEA) del $29,6 \%$, seguido por Perú con el $24,6 \%$, Chile con el $23,8 \%$ y por último Colombia con el 18,7\%. (Lasio, et al., 2018).

Dentro de nuestro país en el mismo año se ha encuestado a 2060 personas con la finalidad de identificar por zonas, regiones, edad y sexo a los emprendedores, de las cuales el $61,34 \%$ pertenecen a la zona urbana, el $38,6 \%$ a la zona rural, con un promedio de edad de 36,24 años, identificando por regiones, la Costa tiene el 49,95\%, seguido por la Sierra con el $44,72 \%$, finalmente la Amazonia con el $5,33 \%$ y por último se tiene que las mujeres son más emprendedoras con el 50,09\%, mientras el hombre es menos emprendedor con el 49,1\% (Lasio, et al., 2018).

En el país la fresa es uno de los Emprendimientos más notables, su producción está distribuida de la siguiente manera: el $50 \%$ proviene de la provincia de Pichincha, luego le sigue la provincia de Tungurahua con el $20 \%$ y finalmente el $30 \%$ está dividida entre las provincias de Chimborazo, Cotopaxi, Azuay y una parte de Imbabura (Rodas \& Tituana, 2012).

En la Provincia de Cañar la fresa se está promoviendo por agricultores y Emprendedores.

Cañar es una provincia ubicada al sur del país, con una extensión de $3908 \mathrm{~km}$, cuenta con 225.184 habientes según (INEC, 2010), está limitada al norte Con Chimborazo, al sur con Azuay, al oeste con Guayas y al este con Morona Santiago, está conformada por 7 cantones, Azogues, Biblián, Cañar, La troncal, Déleg, El tambo y Suscal. Aparte de la fresa han surgido varios Emprendimientos, en la ganadería, la crianza del ganado para 
Segundo Noé Quizhpilema-Acero; Ana Gabriela Hinojosa-Caballero; Renán Teodoro Rodríguez-Pillaga; Juan Edmundo Álvarez-Gavilanes

la producción de la carne y la leche. En la agricultura se cultiva el Maíz, la Patata, la Cebada, el Trigo, el Haba y la Arveja, también se construyen invernaderos para el cultivo de Tomate, Babaco.

La fresa es uno de los Emprendimientos más relevantes de los últimos años, gracias a la producción de la fresa 15 familias del cantón Cañar de diferentes comunidades (Quilloac, San Antonio, La posta, San Pedro y Nar) se ha visto beneficiadas ya que la producción genera ingresos que ayudan el sustento de sus familias (El telégrafo, 2015). Sin embargo, la comercialización se desarrolla solo en el área del medio local lo cual limita los ingresos. Esto se podría mejorar buscando nuevos canales de negocio, innovando, con un valor agregado, dando una nueva imagen al producto y así aumentar las utilidades. Este

problema se mantiene presente por la deficiencia en temas como: Administración, financiación, marketing, producción, mercadeo y ventas. Por tal motivo se da la necesidad de desarrollar un diagnóstico en los emprendimientos de la producción de la fresa del cantón Cañar con el objetivo de identificar los principales problemas y así elevar las utilidades.

\section{Referencial teórico}

Aplicación del diagnóstico en los Emprendimientos con el propósito de solucionar dificultades que permitan alcanzar su desarrollo empresarial

El Emprendimiento es un fenómeno que no es nuevo pero su incorporación es reciente en políticas y en programas de fomento social, así también en los países desarrollados. Por otro lado el Emprendimiento es un tema poco analizado por tal razón la diversificación de su significado. A lo largo de la historia siempre han existido personas que se les ha considerado emprendedores, por la preocupación de los problemas de los individuos y la sociedad, sin ningún interés personal, de la misma forma los grupos religiosos desde hace algún tiempo vienen desarrollando actividades en Bienestar de la sociedad, 
Segundo Noé Quizhpilema-Acero; Ana Gabriela Hinojosa-Caballero; Renán Teodoro Rodríguez-Pillaga; Juan Edmundo Álvarez-Gavilanes

actividades que paulatinamente han ido adoptando las instituciones públicas y organizaciones (Vidal, et al., 2019).

En si un emprendedor es la persona que lidera e identifica el problema social que to aborda, busca sus soluciones y la difunde con el propósito de beneficiar a la sociedad, el Emprendimiento es una forma de organizar recursos con el objetivo principal de un crecimiento económico generando un impacto positivo, además genera empresas cuyo objetivo principal es satisfacer las necesidades de la sociedad y su estabilidad económica, su desarrollo se basa en la trasformación de la economía, la sociedad y la cultura (Fundación Acción contra el Hambre, 2017).

El Emprendimiento es una vía principal que genera utilidades y riquezas por ende contribuye al desarrollo de la sociedad. Una persona o la sociedad analítica es capaz de generar riquezas, utilizando sus ventajas intelectuales personales, herramientas y recursos que se encuentran a su alrededor generando productos que impacten al medio, a esto se lo conoce como empresas generadoras de riquezas (Riveros, 2010). Por lo tanto, un Emprendimiento genera una empresa que es gestionada por un líder o emprendedor con el objetivo de generar un impacto social, operando en el mercado con bienes y servicios de un modo innovador, para alcanzar sus metas de una manera responsable y trasparente involucrando a trabajadores, clientes y grupos de interés (Fundación Acción contra el Hambre, 2017).

Por lo tanto, todas las empresas sin importar que sus actividades o procesos se desarrollen con normalidad. Se debe efectuar un diagnóstico de un periodo tiempo (mensual, semestral o anual) para establecer puntos, donde demuestren debilidad y fortalecerlos. Las anomalías pueden presentarse por diferentes circunstancias como: mala gestión, inadecuada planificación, una cultura y organización mala, productividad inadecuada, falta innovación, clientes insatisfechos (Reyes, et al., 2020). 
Segundo Noé Quizhpilema-Acero; Ana Gabriela Hinojosa-Caballero; Renán Teodoro Rodríguez-Pillaga; Juan Edmundo Álvarez-Gavilanes

Entonces si se necesita conocer cuál es la situación actual de una empresa u organización se debe efectuar un diagnóstico que contribuya identificar cuáles son las causas principales que impiden crecer. El diagnóstico empresarial es una de las herramientas más utilizadas y sobre todo eficaz para este tipo de situación. Gracias a esta herramienta se puede encontrar los problemas de raíz, en cada una de las áreas, para de esta forma desarrollar estrategias que contribuyan a eliminarlos y así apoyen a que la empresa recupere su estado natural o en su defecto pueda crecer (Nuñis, 2017). La aplicación del diagnóstico se puede efectuar de 2 maneras generales. El diagnóstico rutinario y la evaluación final. El rutinario se lo aplica durante el desarrollo de un proceso, con el objetivo de efectuar correcciones para evitar que el problema se agrave, por otro lado la evaluación final se lo aplica cuando el plan ha terminado. El objetivo de la evaluación final, es comprobar si el resultado alcanzado, es lo que ese esperaba, así de este modo confirmar la estrategia desarrollada (Montilla-Galvis, 2004).

\section{Tipos de diagnósticos}

En esta fase del proceso se define la manera de cómo se va a recolectar la información del tema en específico a estudiar tomando en cuenta, tres actividades principales, actividades que contribuirán en la planificación, en el establecimiento de estrategias para llegar a los objetivos y el tipo de diagnóstico que permitirá determinar las herramientas, los recursos, el tiempo y la metodología para la recolección de información necesaria para el desarrollo de los procesos a través de los objetivos establecidos (PadillaCampoverde, et al., 2019).

Diagnóstico sintomático: Este diagnóstico se basa en problemas presentados por el listado de síntomas de la empresa. Esta información resulta insuficiente ya que no tiene todos los detalles del problema, como las causas principales y sus dependencias; por tal razón no se toma como un punto de referencia confiable para la búsqueda de una solución, se trata sobre un diagnóstico habitual de los que comúnmente se lo efectúan a 
Segundo Noé Quizhpilema-Acero; Ana Gabriela Hinojosa-Caballero; Renán Teodoro Rodríguez-Pillaga; Juan Edmundo Álvarez-Gavilanes

través de una serie de pregunta referentes a las funciones, estructuras de la organización, procesos, productos y servicios de la empresa. Cada empresa tiene su propio departamento de consultoría con su propio listado de preguntas para diagnosticar la calidad de servicio.

Diagnóstico etiológico: Este tipo de diagnóstico se basa en la causa del problema de la empresa. Su descripción se refiere a una comparación entre el valor nominal establecido de la empresa y el valor actual, basándose en factores causales de las deficiencias, por tal motivo ofrece un punto de partida confiable para la elaboración de un plan de soluciones. Pero todo este proceso no es suficiente por cuanto no permite identificar la variación que hay entre la relación causa y efecto, ni tampoco permite identificar los diferentes procesos de retroalimentación, lo que impide comprender completamente el problema. Por esta razón antes de poner en práctica la solución se debe simular.

Diagnóstico funcional: Es este caso se refiere en efectuar el diagnóstico a través de la acción de caracterizar un problema establecido por los síntomas que presenta, tomando en cuenta el factor crítico (factor variable independiente que disminuye la desviación entre el valor actual y el valor nominal) y las interdependencias utilizables entre todos los elementos que intervienen. Este tipo diagnostico distingue toda clase de causas que ocasionan las dificultades o también conocido como el "factor crítico". En este caso se debe utilizar técnicas de análisis de sistemas (Maqueda \& Llaguno, 1995).

Diagnóstico cultural: Es un tipo de análisis evalúa la forma de vida de una comunidad, sus hábitos, actitudes, tradiciones, virtudes y sus defectos, que son dadas a conocer por las autoridades, en las juntas, en un lugar determinado, además permiten participar en la comunidad en diferentes actividades tradicionales que están constituidos por varios campos como: ceremonias ancestrales, música, danzas y gastronomía. Para el análisis se recomienda efectuar a través de entrevistas individuales y sobre todo desarrollar una observación de sus estilos de vida (Rodríguez-Piña \& Peña-Domínguez, 2008). 
Segundo Noé Quizhpilema-Acero; Ana Gabriela Hinojosa-Caballero; Renán Teodoro Rodríguez-Pillaga; Juan Edmundo Álvarez-Gavilanes

Diagnóstico tecnológico: Es el diagnóstico que propone a las empresas acciones y objetivos concretos en la tecnología, como por ejemplo la creación de nuevos productos, la adopción de nuevos procesos, proyectos de investigación, con el fin de desarrollar actividades de innovación y fomentar la participación en programas tecnológicos en las empresas extranjeras, conjuntamente con la ayuda de otros países de la comunidad. La metodología utilizada son las siguientes:

1. Potencial de investigación y desarrollo de la empresa

2. Capacidad de apertura

3. Capacidad de gestión

4. Posibilidad de integración a las actividades innovadoras en programas de la comunidad (López, 2015).

Diagnóstico estratégico: En este tipo de diagnóstico nos permite dar una orientación adecuada a la empresa, sus características principales son: El periodo, se realiza cada cierto tiempo (mensual, semestral, anual), además se debe tomar en cuanta, las partes más importantes de las empresas, mediante un modelo de estratégico.

Periodo, en esta etapa se compara la eficiencia actual con años anteriores o con procesos anteriores para así establecer debilidades de la empresa y corregirlas a tiempo.

Áreas de las empresas, se basa en el análisis del organigrama de la institución, donde se identifican las áreas de mayor debilidad.

1. Estratégico, el proceso que se debe llevar cabo es: Permanecía, es la intención de la empresa de mantenerse funcionando durante mucho tiempo.

2. Visualización a largo plazo, es una perspectiva que se tiene de la empresa hacía el futuro para buscar estrategias que mantengan en la elite empresarial.

3. Planteamientos de objetivos, se desarrollan para que una organización se mantenga en los primeros niveles, durante mucho tiempo.

4. Modelos estratégicos, son las vías principales para llegar a los objetivos. 
Segundo Noé Quizhpilema-Acero; Ana Gabriela Hinojosa-Caballero; Renán Teodoro Rodríguez-Pillaga; Juan Edmundo Álvarez-Gavilanes

5. Diagnóstico, es una evaluación para saber si se están aplicando adecuadamente las estrategias planteadas por la empresa para llegar a los objetivos (Rosas, 2012). Diagnóstico empresarial: Es el diagnóstico que se centra en el mundo empresarial, este término hace referencia a una serie de actividades que se lleva a cabo para saber cuál es la situación actual de la empresa y establecer cuales son factores principales que impiden el desarrollo de la empresa. Dentro del mundo empresarial existes diversos tipos de diagnósticos para las empresas, en el cual; algunos se dedican analizar únicamente de una parte especifica como puede ser el desarrollo de comercialización, producción, mercado o marketing etc. Este diagnóstico permite conocer todos los detalles de la empresa, mediante metodologías específicas lo que hace de esta una herramienta indispensable y confiable. Dentro de diagnóstico empresarial existen dos tipos de diagnósticos fundamentales:

Diagnósticos integrales, Son un conjunto de varios tipos de diagnósticos, que son principalmente conocidos porque son variables aplicables, en diferentes campos de una empresa. Por ejemplo el análisis de competitividad que existe entre las asociaciones. Es un estudio que permite evaluar mediante la matriz Foda las ventajas y desventajas, internas y externas que posee la empresa. Otro ejemplo son las técnicas estratégicas, que se dedica a la investigación de las políticas personales, etc. Todo este trabajo lo desarrolla un consultor o alguna persona responsable del diagnóstico que lo efectúa en base evaluaciones y diferentes consideraciones.

Diagnósticos específicos, este tipo de diagnóstico es preciso y concreto. Es conocido principalmente por concentrarse en procesos más específicos como; en los diferentes aspectos relacionados con el mercado, en aspectos de estados financieros o los diferentes procesos de gestión como los inventarios, productividad, red de ventas, y cualquier otro proceso que se vincule con la empresa. 
Segundo Noé Quizhpilema-Acero; Ana Gabriela Hinojosa-Caballero; Renán Teodoro Rodríguez-Pillaga; Juan Edmundo Álvarez-Gavilanes

\section{Pasos para efectuar un diagnóstico empresarial adecuado y eficaz}

Dependiendo del tipo o campo en el que se pretenda aplicar el diagnóstico integral o específico, además, se debe tener en cuenta cuatro específicos procesos que son:

Evaluación, Estableceremos una lista de preguntas que permitirá efectuar el diagnóstico, y así definir la situación actual de la empresa, concentrando en áreas más importantes como: consumidores, calidad, compras, finanzas, procesos de producción, etc.

Visión detallada o concreta, En esta parte del proceso es cuando la persona o grupo de personas encargadas del diagnóstico, recolectan toda la información posible acerca de la situación actual de la empresa, institución, organización o algún tema en concreto. Esta parte del diagnostico se desarrolla a través de la información que otorguen los registros, gráficos y entrevistas $u$ otros recursos recolectados que permiten conocer de primera mano la situación actual del apartado o algún tema en específico.

Cálculos, En estas instancias del proceso y con toda la información recopilada en la fase anterior del diagnóstico, es el momento o la fase donde se establece en qué situación se encuentra la empresa, organización o algún tema en específico que se estudie y además permite saber el nivel de la organización, de acuerdo con la lista que se haya pretendido establecer.

Conclusiones, En esta etapa se analiza toda la información recolectada, se estudia y se evalúan los resultados obtenidos, así se puede identificar las causas de los problemas y mediante lo que se establezca y se puede formular soluciones para los parámetros anteriormente identificados que están impidiendo alcázar los objetivos de la empresa. 
Segundo Noé Quizhpilema-Acero; Ana Gabriela Hinojosa-Caballero; Renán Teodoro Rodríguez-Pillaga; Juan Edmundo Álvarez-Gavilanes

\section{Perspectivas de un diagnóstico empresarial}

El diagnóstico empresarial posee una parte fundamental que son las perspectivas, en esta parte se anunciará 4 apartados principales que se detallaran a continuación.

1. Financiera, Estudia y analiza todo su departamento administrativo y la gestión financiera de la empresa, mediante la revisión y evaluación de sus inversiones, fuentes de financiación y entre otras, para conocer el estado financiero.

2. Clientes, Desde esta parte se analiza el comportamiento del personal laboral, respecto a la relación y trato con los clientes.

3. Procesos internos, En esta parte del proceso se realiza principalmente la evaluación de los procesos de producción, logística, compras, etc. Analizando su productividad, calidad y su eficacia.

4. Talento humano y restos de recursos, Evalúa la capacidad del personal activo de la empresa, para alcanzar el alto nivel que se requiere para la excelencia, además evalúa los diferentes canales para desarrollar los diferentes procesos (Nuñis, 2017). 
Segundo Noé Quizhpilema-Acero; Ana Gabriela Hinojosa-Caballero; Renán Teodoro Rodríguez-Pillaga; Juan Edmundo Álvarez-Gavilanes

\section{Áreas de análisis del diagnóstico empresarial}

Cada una de las empresas posee sus propias áreas de trabajo, en este caso se analizará las siguientes áreas: producción, financiera, talento humano y ventas donde se procederá a tabular los datos recolectados y así establecer la situación actual de la empresa.

Área de producción:

\section{Tabla 1.}

Volúmenes de producción de la fresa.

\begin{tabular}{lcc}
\hline Tendencia & Calificación & Semanales \\
\hline Muy bueno & 10 & $31-40$ \\
Bueno & 7 & $21-30$ \\
Malo & 5 & $11-20$ \\
Muy malo & 3 & $6-10$ \\
Pésimo & 2 & $0-5$ \\
\hline
\end{tabular}

Área ventas:

Tabla 2.

Promedio de ventas realizadas.

\begin{tabular}{lcc}
\hline Tendencia & Calificación & Porcentaje \\
\hline Muy bueno & 10 & $(90-100) \%$ \\
Bueno & 7 & $(70-89) \%$ \\
Malo & 5 & $(36-69) \%$ \\
Muy malo & 3 & $(16-35) \%$ \\
Pésimo & 2 & $(0-15) \%$ \\
\hline
\end{tabular}


Segundo Noé Quizhpilema-Acero; Ana Gabriela Hinojosa-Caballero; Renán Teodoro Rodríguez-Pillaga; Juan Edmundo Álvarez-Gavilanes

Área de finanzas:

\section{Tabla 3.}

Rentabilidad patrimonial.

\begin{tabular}{lcc}
\hline Tendencia & Calificación & Porcentaje \\
\hline Muy bueno & 10 & $(90-100) \%$ \\
Bueno & 7 & $(70-89) \%$ \\
Malo & 5 & $(36-69) \%$ \\
Muy malo & 3 & $(16-35) \%$ \\
Pésimo & 2 & $(0-15) \%$ \\
\hline
\end{tabular}

Área de talento humano:

\section{Tabla 4.}

Eficiencia laboral.

\begin{tabular}{lcc}
\hline Tendencia & Calificación & Porcentaje \\
\hline Muy bueno & 10 & $(90-100) \%$ \\
Bueno & 7 & $(70-89) \%$ \\
Malo & 5 & $(36-69) \%$ \\
Muy malo & 3 & $(16-35) \%$ \\
Pésimo & 2 & $(0-15) \%$ \\
\hline
\end{tabular}

El resultado de cada una de las áreas será el producto de la tabulación de múltiples indicadores que dará un resultado final y determinará la situación actual de cómo se encuentra cada área, además gracias a la recolección de estos datos y la tabulación se tendrá un promedio final, determinando el estado de la empresa. 
Segundo Noé Quizhpilema-Acero; Ana Gabriela Hinojosa-Caballero; Renán Teodoro Rodríguez-Pillaga; Juan Edmundo Álvarez-Gavilanes

\section{Tabla 5.}

Indicadores de calificación final de la empresa.

\begin{tabular}{lccc}
\hline Tendencia & Indicadores & Calificación & Porcentaje \\
\hline Muy bueno & MB & 10 & $(90-100) \%$ \\
Bueno & $\mathrm{B}$ & 7 & $(70-89) \%$ \\
Malo & $\mathrm{M}$ & 5 & $(50-69) \%$ \\
Muy malo & $\mathrm{MM}$ & 3 & $(30-49) \%$ \\
Pésimo & $\mathrm{P}$ & 2 & $(0-29) \%$ \\
\hline
\end{tabular}

\section{Aplicación del modelo}

Área de producción

Indice de disponibilidad

Es el tiempo disponible en los procesos de producción de una empresa. Su fórmula, Índice de disponibilidad es igual al tiempo de proceso (Es tiempo de ejecución del proceso para la obtención del producto) sobre el tiempo real operativo (Es el tiempo del proceso, incluyendo sus paradas) multiplica por 100 para obtener el porcentaje.

I. de disponibilidad= (Tiempo de proceso $)$ ( $($ Tiempo real operativo) $\times 100 \%$ Índice de producción

Indica a que ritmo se obtiene la producción, además indica si se está cumpliendo con la producción programada.

I. de producción $=($ Unidades reales $) /$ (unidades teóricas) $\times 100 \%$

Índice de calidad 
Segundo Noé Quizhpilema-Acero; Ana Gabriela Hinojosa-Caballero; Renán Teodoro Rodríguez-Pillaga; Juan Edmundo Álvarez-Gavilanes

Uno de los objetivos en una empresa es ofrecer al cliente un producto de calidad, ya que este parámetro influye en gran porcentaje en su imagen, además, este parámetro puede ocasionar la pérdida de los clientes. Este indicador es de gran importancia ya que permite medir la confiabilidad del proceso y establecer el control de calidad. La fórmula para la obtención del índice de calidad es el número de unidades defectuosas sobre el total de unidades producidas; a este resultado lo multiplicamos por 100 y lo restamos $100 \mathrm{el}$ resultado lo obtenemos en porcentaje.

I. de calidad $=($ Unidades defectuosas $) /($ Unidades producida $) \times 100 \%-100 \%$

Índice de efectividad

Es el parámetro que indica el resultado general de la planta o área a partir de los siguientes parámetros: porcentaje de disponibilidad, porcentaje de productividad y el porcentaje de calidad.

I. efectv. $=(\%$ de tiempo disponible $)(\%$ de productividad $)(\%$ de calidad $)$

Área de ventas

Nivel de desempeño de ventas establecidas

Las ventas, es el objetivo principal de la empresa o en otras palabras es la principal fuente de supervivencia de un negocio que se lleva a cabo a través de un ingreso neto, este indicador mide el grado de ventas establecidas en un periodo determinado.

I. de cumplimiento de ventas $=($ Ventas netas $) /($ Ventas establecidas $) \times 100 \%$ Área de finanzas

Total de deudas de la empresa

En una empresa, dentro del área de finanzas, es muy importante establecer el nivel de deudas que se tiene. De acuerdo con este parámetro se puede saber cuál es la capacidad que se tiene para pagar las deudas, a la vez se puede saber cuál es el número de acciones que tiene cada inversionista. El factor también permite saber si la organización se encuentra en un grado bajo, medio o alto de riesgo, lo que conlleva a concluir a mayor deuda mayor probabilidad de poner en riesgo a la empresa. Además, proporciona una 
Segundo Noé Quizhpilema-Acero; Ana Gabriela Hinojosa-Caballero; Renán Teodoro Rodríguez-Pillaga; Juan Edmundo Álvarez-Gavilanes

información clara acerca de la calidad de confiabilidad que se tiene sobre los pasivos. Una nota importante en esta sección es que las deudas que se generen tiene que ser menor a la posibilidad de pago.

Endeudamiento. $\mathrm{T}=($ Pasivo. T)/ (Activo. T) $(100 \%)$

Cobertura de interés

Es la posibilidad que la asociación tiene para pagar los interés establecido. Permite medir la incidencia de los gastos financieros sobre la utilidad que ha adquirido las operaciones de la empresa. El resultado obtenido indica cuantas veces la utilidad adquirida puede pagar el interés.

Cobertura de interés $=$ UAll/ Intereses finiquitados

Rentabilidad neta

Permite saber el \% neto de utilidad que se adquiere de una inversión.

Rentabilidad. $\mathrm{N}=$ (Utilidad neta después de los impuestos)/ (Patrimonio neto) $\times 100 \%$

Ejecución presupuestal de gastos

Es una comparación entre los egresos reales y los presupuestados.

Ejecución presupuestal de egresos $=(T$. de egresos reales $) /(T$. de egresos presupuestados) $\times 100 \%$

\section{Área de Talento Humano}

Eficiencia laboral

Un factor importante en una organización, es establecer un salario acorde a las escalas establecidas para empresas del mismo tamaño y del mimo sector. El salario no se puede alejar de las escalas establecidas. Gracias a estos parámetros se puede medir, la eficiencia laboral, además detecta gastos innecesarios que no tienen relación a las actividades laborales (Montilla, 2004).

Eficiencia laboral= (Salarios y prestaciones sociales $) /($ Ingresos netos) $\times 100 \%$ 
Segundo Noé Quizhpilema-Acero; Ana Gabriela Hinojosa-Caballero; Renán Teodoro Rodríguez-Pillaga; Juan Edmundo Álvarez-Gavilanes

\section{METODOLOGÍA}

La investigación se fundamentó centralmente en el paradigma positivista de observación, medición y verificación, con un enfoque cuantitativo y un tipo descriptivo de diseño de campo no experimental (Aldana-Zavala, 2019), lo cual permitió estudiar desde una óptica documental - bibliográfica el conocimiento teórico referente a las variables de estudio, lo cual permitió a los investigadores investigar en una fase asincrónica con la finalidad de comparar en una etapa sincrónica con los datos recopilados, comparando así el comportamiento en la realidad, permitiendo analizar el objeto observado y medido a través de la encuesta y cuestionario, siendo procesado a un universo poblacional de 6 emprendedores del cantón Cañar, ciudad de Cañar, de la provincia Cañar-Ecuador, gestionando el análisis estadistico desde una perspectiva descriptiva.

\section{RESULTADOS}

Los resultados alcanzados a través de las encuestas y entrevistas realizadas a los emprendedores, están representadas en la siguiente tabla: 
Revista Arbitrada Interdisciplinaria KOINONIA

Año 2020. Vol V. №3. Especial: Administración

Hecho el depósito de Ley: FA2016000010

ISSN: 2542-3088

FUNDACIÓN KOINONIA (F.K). Santa Ana de Coro. Venezuela.

Segundo Noé Quizhpilema-Acero; Ana Gabriela Hinojosa-Caballero; Renán Teodoro Rodríguez-Pillaga; Juan Edmundo Álvarez-Gavilanes

Tabla 6 .

Resultados de las encuestas realizadas a los emprendedores.

\begin{tabular}{|c|c|c|c|c|c|c|c|c|c|c|c|c|c|c|c|}
\hline \multirow{2}{*}{ Áreas } & \multirow{2}{*}{ Preguntas } & \multirow{2}{*}{ Unidad } & \multicolumn{6}{|c|}{ Emprendimientos } & \multirow{2}{*}{ Total } & \multirow{2}{*}{ Promedio } & \multirow{2}{*}{\multicolumn{3}{|c|}{ Porcentaje }} & \multirow{2}{*}{\multicolumn{2}{|c|}{ Total \% }} \\
\hline & & & 1 & 2 & 3 & 4 & 5 & 6 & & & & & & & \\
\hline \multirow{4}{*}{ 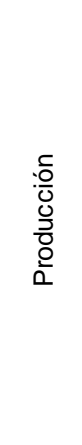 } & $\begin{array}{l}\text { ¿Cuál es el tiempo de } \\
\text { proceso para la } \\
\text { producción de la fresa? }\end{array}$ & Meses & 4 & 4 & 4 & 4 & 4 & 4 & 24 & 4 & 100 & 0 & 0 & 0 & 100 \\
\hline & $\begin{array}{l}\text { ¿Cuál es tiempo neto } \\
\text { operativo para la } \\
\text { producción de la fresa? }\end{array}$ & Meses & 4,25 & 4,25 & 4,25 & 4,25 & 4,25 & 3,75 & 25 & 4,17 & 83,3 & 16,7 & 0 & 0 & 100 \\
\hline & $\begin{array}{l}\text { ¿Cuántas unidades } \\
\text { produce el cultivo de } \\
\text { fresa a la semana? }\end{array}$ & Gavetas & 14 & 14 & 13 & 13 & 13 & 13 & 80 & 13,33 & 33,34 & 66,66 & 0 & 0 & 100 \\
\hline & $\begin{array}{l}\text { ¿Cuántas unidades de } \\
\text { fresa son defectuosas? }\end{array}$ & Gavetas & 5 & 4 & 4 & 3 & 2 & 2 & 20 & 3,33 & 16,66 & 33,34 & 16,67 & 33,33 & 100 \\
\hline \multirow{2}{*}{ 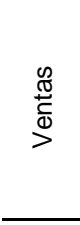 } & $\begin{array}{l}\text { ¿Cuántas gavetas de } \\
\text { fresa salen para la venta } \\
\text { a la semana? }\end{array}$ & Gavetas & 9 & 10 & 9 & 10 & 11 & 11 & 60 & 10 & 33,35 & 33,33 & 33,32 & 0 & 100 \\
\hline & $\begin{array}{l}\text { De las unidades } \\
\text { sacadas a la venta } \\
\text { ¿cuántas se venden? }\end{array}$ & Gavetas & 8,5 & 9,3 & 8,5 & 9,3 & 10,4 & 10,4 & 56,46 & 9,41 & 33,35 & 33,34 & 33,31 & 0 & 100 \\
\hline \multirow{6}{*}{$\begin{array}{l}\mathbb{N} \\
\stackrel{N}{N} \\
\stackrel{\mathbb{T}}{=} \\
i=\end{array}$} & $\begin{array}{l}\text { ¿Cuál es el total de } \\
\text { pasivos que tiene el } \\
\text { Emprendimiento? }\end{array}$ & Dólares & 2500 & 2500 & 2000 & 2000 & 2000 & 2000 & 13000 & 2166,67 & 33,34 & 66,66 & 0 & 0 & 100 \\
\hline & $\begin{array}{l}\text { ¿Cuál es el total de } \\
\text { activos que tiene el } \\
\text { Emprendimiento? }\end{array}$ & Dólares & 3000 & 3000 & 3000 & 2000 & 2000 & 2000 & 15000 & 2500 & 50,02 & 49,98 & 0 & 0 & 100 \\
\hline & $\begin{array}{l}\text { ¿Cuánto es la utilidad } \\
\text { adquirida de la empresa } \\
\text { al mes? }\end{array}$ & Dólares & 600 & 660 & 540 & 660 & 540 & 600 & 3600 & 600 & 33,35 & 33,33 & 33,32 & 0 & 100 \\
\hline & $\begin{array}{l}\text { ¿Cuánto es el interés } \\
\text { pagado mensualmente? }\end{array}$ & Dólares & 185 & 188 & 187,5 & 188 & 188 & 188 & 1123 & 187,15 & 16,67 & 83,33 & 0 & 0 & 100 \\
\hline & $\begin{array}{l}\text { ¿Cuánto son los gastos } \\
\text { para la producción de la } \\
\text { fresa al mes? }\end{array}$ & Dólares & 25 & 40 & 20 & 40 & 25 & 30 & 180 & 30 & 16,66 & 33,34 & 16,67 & 33,33 & 100 \\
\hline & $\begin{array}{l}\text { ¿Cuánto son los gastos } \\
\text { presupuestados en la } \\
\text { producción al mes? }\end{array}$ & Dólares & 30 & 45 & 30 & 40 & 30 & 30 & 205 & 34,17 & 16,67 & 16,65 & 66,68 & 0 & 100 \\
\hline \multirow{2}{*}{ 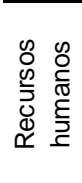 } & $\begin{array}{l}\text { ¿Cuántas personas } \\
\text { laboran en el } \\
\text { Emprendimiento? }\end{array}$ & Personal & 2 & 2 & 1 & 3 & 2 & 2 & 12 & 2 & 66,66 & 16,68 & 16,66 & 0 & 100 \\
\hline & $\begin{array}{l}\text { ¿Cuántas horas laboran } \\
\text { cada personal al mes? }\end{array}$ & Horas & 31 & 32 & 28 & 32 & 29 & 28 & 180 & 30 & 33,34 & 33,33 & 16,67 & 16,66 & 100 \\
\hline
\end{tabular}

Fuente: Investigadores. 
Segundo Noé Quizhpilema-Acero; Ana Gabriela Hinojosa-Caballero; Renán Teodoro Rodríguez-Pillaga; Juan Edmundo Álvarez-Gavilanes

\section{Aplicación en el modelo para determinar estado de las áreas}

Para conocer la situación actual de las micro-empresas, la investigación se basa en la aplicación de fórmulas en diferentes áreas, para lo cual se necesita conocer varios datos que están representadas en la tabla anterior que posteriormente son aplicadas en las fórmulas, con el objetivo obtener una apreciación más exacta de la situación actual de los Emprendimientos.

Área de producción

Indice de disponibilidad

- - . de disponibilidad= $($ Tiempo de proceso $) /$ (Tiempo neto operativo) $\times 100 \%$

- - . de disponibilidad $=(4$ meses $) /(4,16$ meses $) \times 100 \%$

- . de disponibilidad $=96,15 \%$

Índice de producción

-I. de producción $=($ Unidades reales $) /$ (unidades teóricas) $\times 100 \%$

-I. de producción $=10 / 13 \times 100 \%$

-I. de producción=76,09\%

Índice de calidad

- - de calidad $=$ (Unidades defectuosas) $/$ (Unidades producida) $\times 100 \%-100 \%$

-I. de calidad $=3,33 / 13,33 \times 100 \%-100 \%$

-I. de calidad $=75,01 \%$

Índice de efectividad

-I. efectv. $=((\%$ de tiempo disponible $)+(\%$ de productividad $)+(\%$ de calidad $)) / 3$

- -. efectv. $=((96,15 \%)+(76,09 \%)+(75,01 \%)) / 3$

- I. efectividad $=82,45 \%$

Área de ventas

Nivel de desempeño de ventas establecidas

- I. de cumplimiento de ventas $=($ Ventas netas $) /($ Ventas establecidas $) \times 100 \%$ 
Segundo Noé Quizhpilema-Acero; Ana Gabriela Hinojosa-Caballero; Renán Teodoro Rodríguez-Pillaga; Juan Edmundo Álvarez-Gavilanes

- I. de cumplimiento de ventas $=9,41 / 10 \times 100 \%$

-I. de cumplimiento de ventas $=94,1 \%$

Área de finanzas

Total de deudas de la empresa

-Endeudamiento. $\mathrm{T}=$ (Pasivo. T)/ (Activo. T) (100\%)

-Endeudamiento. $\mathrm{T}=2116,67$ / 2500 (100\%)

-Endeudamiento. $\mathrm{T}=84,66 \%$

Cobertura de interes

-Cobertura de interés $=$ UAII/(Intereses finiquitados)

-Cobertura de interés $=600 \$ / 187,15 \$$

-Cobertura de interés $=3,20$ veces

Ejecución presupuestal de gastos

-Ejecución presupuestal de egresos $=(T$. de egresos reales $) /(T$. de egresos presupuestados) $\times 100 \%$

-Ejecución presupuestal de egresos $=30 \$ / 34,17 \$ \times 100 \%$

-Ejecución presupuestal de egresos=87,79\%

Rentabilidad neta

-Rentabilidad. $\mathrm{N}=$ (Utilidad neta después de los impuestos)/ (Patrimonio neto) $\mathrm{x}$ $100 \%$

-Rentabilidad. $\mathrm{N}=570 \$ / 2500 \$ \times 100 \%$

-Rentabilidad. $\mathrm{N}=0,228 \times 100 \%=22,8 \%$

-Eficiencia del área de finanzas $=77,2 \%$

Área de Talento Humano

Eficiencia laboral

- Ingreso neto $=570 \$$

-Salario $=$ (Ingreso neto)/ (Número de empleados)

-Salario $=570 / 2=285 \$$ 
Segundo Noé Quizhpilema-Acero; Ana Gabriela Hinojosa-Caballero; Renán Teodoro Rodríguez-Pillaga; Juan Edmundo Álvarez-Gavilanes

$-1 \mathrm{mes}=160 \mathrm{~h}$ Laborables

$-(285 \$ \times 160 h) / 30 h=53,43 \$$

-Eficiencia laboral $=53,43 / 285 \times 100 \%$

-Eficiencia laboral $=18,75 \%$

-Eficiencia del talento humano=80,14\%

\section{Presentación de los resultados de los cálculos desarrolladas en las micro- empresas}

\section{Área de producción}

Dentro de esta área se debe establecer varios índices para conocer la situación de cómo se encuentra en la actualidad la producción.

\section{Índice de disponibilidad}

Para establecer el índice de disponibilidad se debe contar con datos importantes como; el tiempo establecido para la producción y el tiempo real de la producción, donde el tiempo establecido para la producción de la fresa es de 4 meses. Mientras que para el tiempo real se tiene que el $83,3 \%$ de encuestados, manifiesta que la producción se obtiene en 4,25 meses, y el 16,7\% indica que la producción se da 3,75 meses, obteniendo un promedio de 4,16 meses para la producción. Con los datos obtenidos se calcula el Índice disponibilidad que es el 96,15\%.

\section{Índice de producción}

Para este índice, se debe conocer primero; las unidades teorías de producción, donde al aplicar las encuestas el $66,66 \%$ manifiestan que se produce 14 gavetas a la semana, mientas que el $33,34 \%$ indican que la producción es de 13 gavetas a la semana, teniendo un promedio de 13,33 gavetas teóricas a la semana. 
Segundo Noé Quizhpilema-Acero; Ana Gabriela Hinojosa-Caballero; Renán Teodoro Rodríguez-Pillaga; Juan Edmundo Álvarez-Gavilanes

Después se debe conocer, las unidades reales de producción, pero antes se debe conocer las unidades de producción defectuosas, donde el 16,66\% manifiesta que 5 son defectuosas, el $33,34 \%$ indica que 4 son defectuosas, el $16,66 \%$ dicen que 3 son defectuosas y por último el 33,32\% manifiestan que 2 son defectuosas, obteniendo un promedio de 3,33 gavetas defectuosas de fresa. Con estos datos obtiene las unidades reales de 10 gavetas a la semana. Finalmente, se procede aplicar en la fórmula donde el índice de producción es de 76,09\%.

\section{Índice de calidad}

Para obtener el índice de calidad se necesita conocer los datos siguientes ; unidades producidas y unidades defectuosas que ya fueron calculados anteriormente y estos datos son; 3,33 y 13,33 gavetas respectivamente que finalmente aplicados en la fórmula se tiene el $75,01 \%$ del índice de calidad de los productos.

\section{Índice de efectividad}

Este índice es el resultado de todo el área de producción para lo cual se tiene en cuenta los tres parámetros calculados anteriormente; índice de disponibilidad, de producción y de calidad que son; $96,15 \%, 76,09 \%$ y $75,01 \%$ respectivamente que aplicados en la fórmula se tiene como resultado del índice de efectividad de la producción de 82,45\%.

Área de ventas.

\section{Índice de cumplimento o desempeño de ventas}

Para definir este índice, se tiene que conocer, las ventas establecidas a la semana o la cantidad de gavetas de fresa que salen a la venta y ventas netas realizadas, donde el $33,35 \%$ de los encuestados manifiestan que salen a la venta 11 gavetas, el 33,33\% indican que salen 10 gavetas y por último el 33,32\% indican que salen 9 gavetas, de estos datos se tiene un promedio de 10 gavetas de fresa que salen para la venta. 


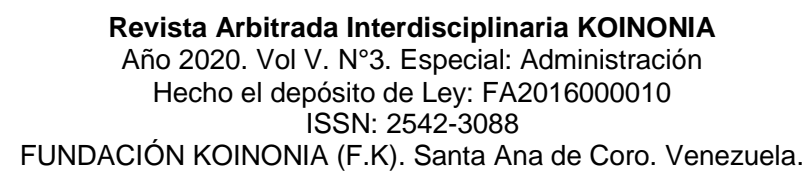

Segundo Noé Quizhpilema-Acero; Ana Gabriela Hinojosa-Caballero; Renán Teodoro Rodríguez-Pillaga; Juan Edmundo Álvarez-Gavilanes

Para conocer el parámetro de las ventas netas realizadas a la semana, se hizo necesario investigar y se obtuvo que el $33,35 \%$ venden 10,43 gavetas, el $33,34 \%$ vende 9,3 gavetas y finamente se tiene que el $33,31 \%$ venden 8,5 gavetas de fresa a la semana, de donde se obtiene un promedio de 9,41 gavetas de fresas vendidas a la semana. Con estos datos se obtiene el índice de ventas de $94,1 \%$.

\section{Área de finanzas}

Total de deudas de la empresa

Dentro de este parámetro, se necesita conocer tanto los pasivos como los activos en su totalidad, para lo cual se investigó y se logró identificar que el 33,34\% tenían en pasivos $2500 \$$ mientras que el $66,66 \%$ tenían $2000 \$$ en pasivos, por lo cual se tiene un promedio de los Emprendimientos en pasivos de $2333,33 \$$.

De la misma forma se hizo necesario averiguar el total de activos que poseen, en el cual el $50,02 \%$ manifiestan que tiene un total de activos de $3000 \$$ y el $49,8 \%$ indican que tienen 2500 en activos, mediante estos datos se establece un promedio total en activos de $2500 \$$. Finalmente, a través de estos datos se obtiene un endeudamiento total de $84,66 \%$.

\section{Cobertura de interés}

Para conocer la cobertura de interés se necesita investigar la utilidad adquirida por los Emprendimientos y también se debe conocer los intereses cancelados al mes, para el primer parámetro se obtuvo que $33,35 \%$ obtiene $660 \$$ de utilidad al mes, el $33,33 \%$ obtiene $600 \$$ al mes y por último se tiene que $33,32 \%$ tiene una utilidad de $540 \$$ al mes, dando un promedio $600 \$$ de utilidad al mes en los Emprendimientos de la fresa.

El dato siguiente que se debe tener en cuenta, son los intereses que cancelaban al mes los emprendedores, mediante la encuesta se tiene que el $83,33 \%$ cancelaba un monto de $187,5 \$$ y el $16,67 \%$ cancelaba un valor de $185,4 \$$, con estos datos se asume un promedio de los intereses pagados al mes de $187,15 \$$. Una vez obtenido los datos se 


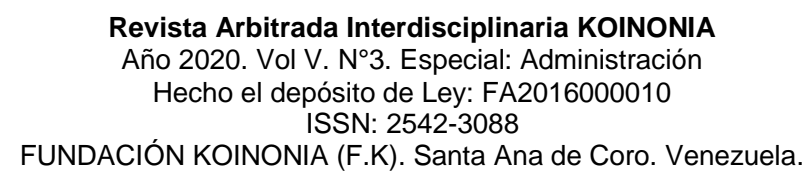

Segundo Noé Quizhpilema-Acero; Ana Gabriela Hinojosa-Caballero; Renán Teodoro Rodríguez-Pillaga; Juan Edmundo Álvarez-Gavilanes

aplican en la fórmula y se obtiene que la cobertura de interés de 3,20 veces esto quiere decir que la utilidad tiene la facultad de cancelar la deuda en 3,20 veces.

\section{Ejecución presupuestal de gastos}

Primeramente en esta parte se debe conocer el total de egresos reales al mes, para lo cual se investigó y se obtuvo que el $33,34 \%$ tienen un egreso de $40 \$$ al mes, mientras que el $16,66 \%$ tiene un gasto de 30 dólares al mes, de la misma forma se tiene que el $33,33 \%$ genera un egreso de $25 \$$ al mes y el 16,67 tiene un gasto de $20 \$$ al mes que finalmente da como un promedio de egresos de $30 \$$ al mes.

En segundo lugar se necesita saber el total de egresos presupuestados al mes, donde el $16,67 \%$ indican que los gastos presupuestados son de $45 \$$, el $16,66 \%$ manifiestan que los gastos son de $40 \$$ y el $66,68 \%$ indican que los gastos son de $30 \$$, a través de estos datos se obtiene el resultado final de los gastos establecidos al mes de $34,17 \$$ y por último se aplica en la fórmula y se obtiene la ejecución de gastos presupuestados de $87,79 \%$.

\section{Rentabilidad neta}

Para conocer la rentabilidad de los Emprendimientos es necesario identifica la utilidad neta y también el patrimonio real, con los datos calculados anteriormente se puede establecer estos parámetros, de donde la utilidad neta es de $570 \$$ al mes y el patrimonio real que es de $2500 \$$, por lo cual al aplicar en la fórmula se tiene un resultado de $22,8 \%$ de rentabilidad.

\section{Recursos humanos}

En el área de recursos humanos primeramente se tiene que investigar cuantas personas se necesitan para una producción en una extensión de terreno de 375 m2 ya que es un dato que se le considera como un punto de referencia para los cálculos, donde el 66,66\% indica que 2 personas son suficientes, el 16,68\% manifiestan que se necesita 3 personas 
Segundo Noé Quizhpilema-Acero; Ana Gabriela Hinojosa-Caballero; Renán Teodoro Rodríguez-Pillaga; Juan Edmundo Álvarez-Gavilanes

y el $16,66 \%$ indican que se necesita una sola persona, con estos datos se obtiene un promedio de 2 personas para la producción.

Además, se investigó cuantas horas laboran al mes cada persona donde se obtuvo que el $33,34 \%$ indican que cada persona trabaja un total de 32 horas, $16,67 \%$ manifiesta que cada empleado trabaja 31 horas, el 16,66\% establece que un empleado trabaja 29 horas y el $33,33 \%$ indican que cada empleado trabaja 28 horas, de estos datos se deduce un promedio de 30 horas laboradas por cada empleado al mes.

Mediante estos datos y con la rentabilidad neta calculada anteriormente se aplica en la fórmula y se obtiene la eficiencia laboral de 18,75\%.

\section{Tabla 7.}

Resultado final de cada una de las áreas de los Emprendimientos.

\begin{tabular}{lcc}
\multicolumn{1}{c}{ Áreas } & $\%$ & Indicadores \\
\hline Productividad & 82,45 & Bueno \\
Ventas & 94,1 & Muy Bueno \\
Finanzas & 77,2 & Bueno \\
Talento Humano & 80,14 & Bueno \\
\hline Total & 333,89 & \\
\hline Promedio & 83,47 & Bueno \\
\hline
\end{tabular}

Fuente: Emprendedores de fresa del cantón Cañar. 
Segundo Noé Quizhpilema-Acero; Ana Gabriela Hinojosa-Caballero; Renán Teodoro Rodríguez-Pillaga; Juan Edmundo Álvarez-Gavilanes

Una vez obtenido los resultados de las diferentes áreas de los Emprendimientos, que se logro a través de las encuestas realizadas a los emprendedores se obtuvo los resultados siguientes siendo estos, el área de ventas con un mayor porcentaje con el 94,1\% catalogado como un nivel Muy bueno por otra parte se tiene el área de finanzas con el porcentaje más bajo de $77,2 \%$ catalogado como bueno, a través de estos resultados se logró determinar la situación actual de los Emprendimientos, ubicándolo en nivel de bueno con un porcentaje de $83,47 \%$.

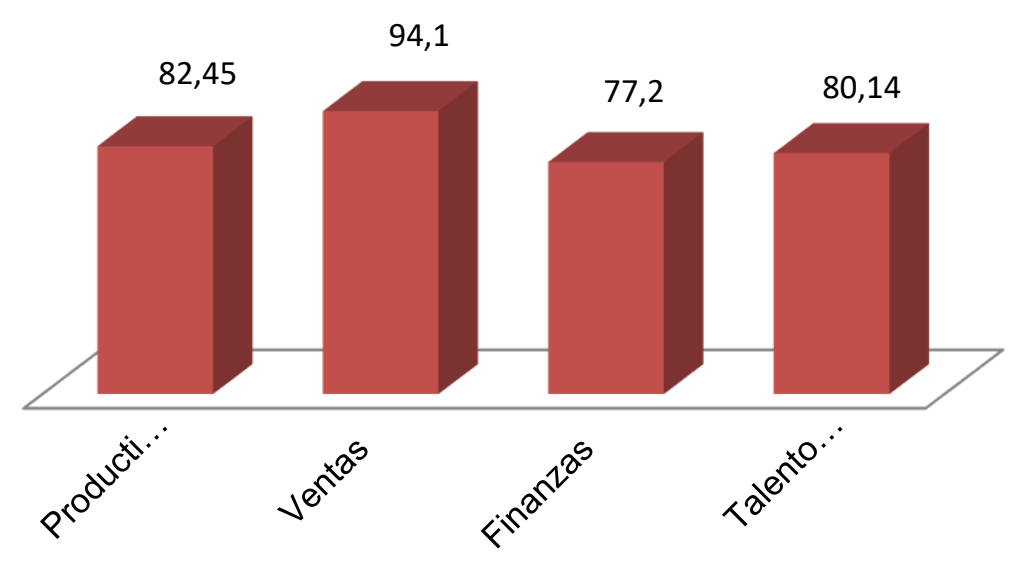

Figura 1. Resultados de cada área de los emprendimientos.

\section{Problemas o puntos críticos identificados en los Emprendimientos de la fresa}

En la investigación se ha utilizado la matriz DAFO para la identificación de los principales problemas de los Emprendimientos de la fresa. Como se sabe la matriz es una herramienta de primer nivel en ámbito empresarial ya que a través de un diagnóstico permite conocer los problemas principales y determinar la situación de la empresa. Está matriz permite tomar decisiones estratégicas oportunas para mejorar en el futuro. (Espinosa, 2013). 
Segundo Noé Quizhpilema-Acero; Ana Gabriela Hinojosa-Caballero; Renán Teodoro Rodríguez-Pillaga; Juan Edmundo Álvarez-Gavilanes

Mediante esta herramienta se ha logrado identificar diferentes problemas, o barreras que impiden el crecimiento y el desarrollo de los Emprendimientos. Las causas identificadas son diversas entre las que se destacan se encuentran las siguientes:

1. Inseguridad para acceder a los financiamientos.

2. Baja iniciativa.

3. Carencias en la investigación de los canales de negocio.

4. Desconocimiento en los procesamientos de los productos.

5. Deficiencia en el área de finanzas.

6. Dificultades de comunicación.

7. Desconocimiento en el área de marketing y publicidad.

8. Falta de conocimientos en la innovación.

Las causas son los responsables de generar los problemas, debilidades o puntos críticos en los Emprendimientos. Los principales problemas o debilidades se encuentran identificados en la siguiente matriz DAFO. 
Segundo Noé Quizhpilema-Acero; Ana Gabriela Hinojosa-Caballero; Renán Teodoro Rodríguez-Pillaga; Juan Edmundo Álvarez-Gavilanes

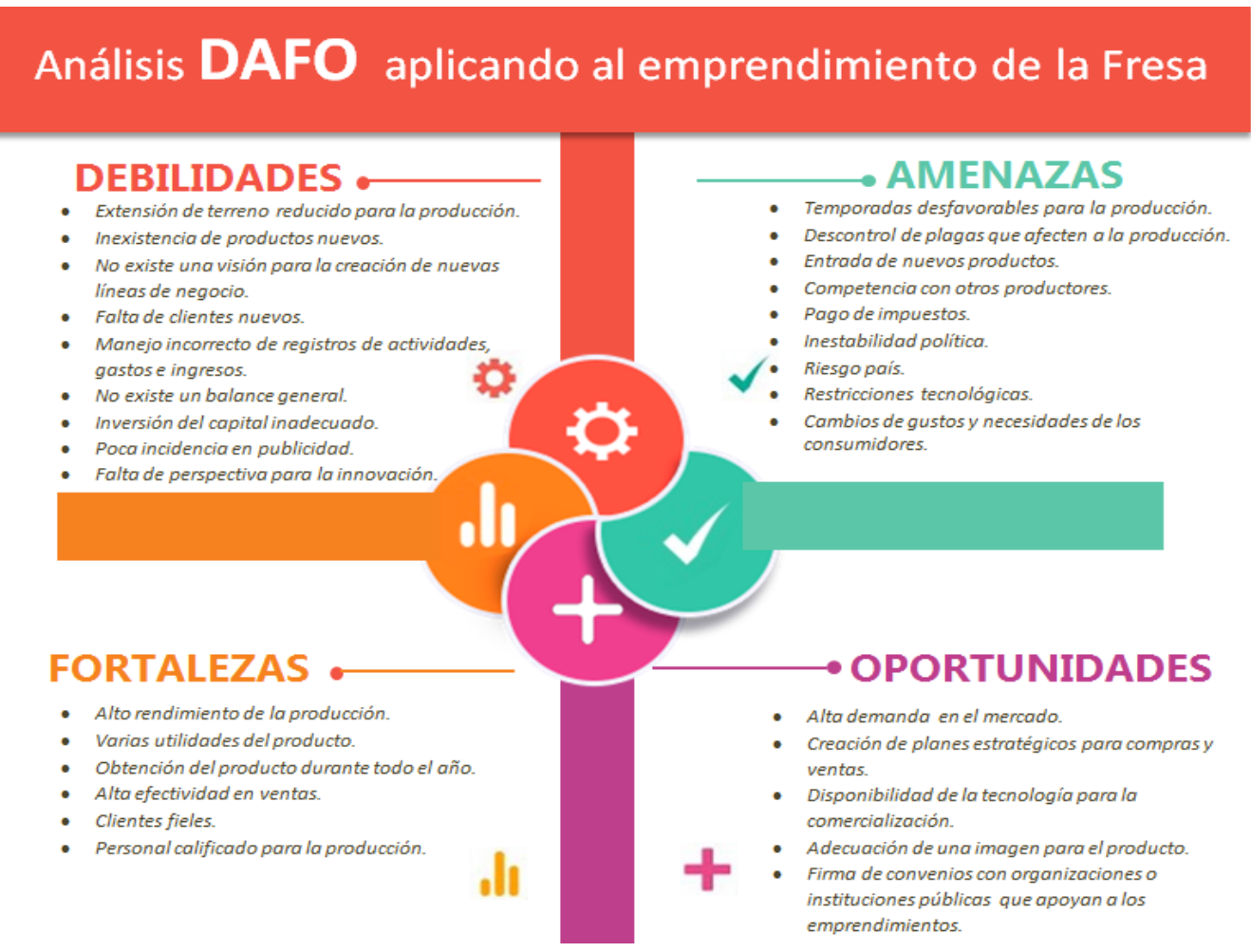

Figura 2. Análisis de la matriz DAFO en los emprendimientos.

A través del estudio realizado se pudo estimar que los Emprendimientos se encuentran en un buen nivel, sin embargo gracias a la matriz DAFO se pudo apreciar que se puede llevar a un nivel de excelencia teniendo en cuenta aspectos relevantes como las varias aplicaciones que tienen los productos razón por lo cual el nivel de ventas es excelente, otro de los aspectos que se debería tener bien identificado es la innovación ya que contribuirá, a comercializar el producto en nuevos canales de negocio pudiendo ser este internacionalmente. 


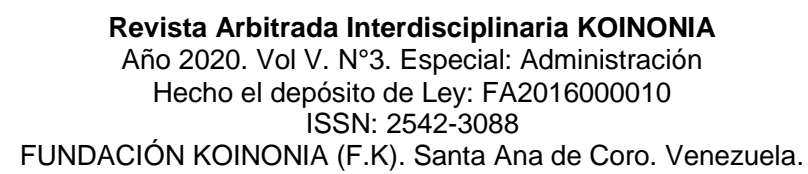

Segundo Noé Quizhpilema-Acero; Ana Gabriela Hinojosa-Caballero; Renán Teodoro Rodríguez-Pillaga; Juan Edmundo Álvarez-Gavilanes

\section{CONCLUSIONES}

Dentro de una empresa u organización es indispensable efectuar un diagnóstico, porque permiten identificar los principales problemas o puntos críticos que impiden el desarrollo y el crecimiento empresarial, además contribuye para que el responsable de la institución tome decisiones para mejorar mediante estrategias basadas en dificultades identificados. El modelo de diagnóstico que se utilizó permitió visualizar el estado actual la organización y de cada una las áreas, siendo así que el área de ventas tiene el porcentaje más alto con un posicionamiento excelente, por otro lado se tiene el área de finanzas de menor porcentaje en posicionamiento bueno, gracias a estas apreciaciones podrán formular planes estratégicos que optimicen los procesos y generen menores costos de producción, contribuyendo en la obtención de productos de calidad.

Además, este modelo de análisis permitió identificar que esta actividad es muy favorable por cuanto el nivel de endeudamiento o la inversión es poca y sobre todo porque sus productos son muy requeridos en el mercado razón por la cual el nivel de ventas es muy alto.

Una de las deficiencias más sobresalientes que se puede resaltar es que los emprendedores se dedican a comercializar el producto solo en el área local, lo cual conlleva a que tengan una visualización muy reducida de la comercialización, además impide que no se tenga una perspectiva para la innovación limitando los ingresos.

El modelo de diagnóstico que se utiliza permite efectuar comparaciones entre distintas áreas de la organización para de esta forma identificar el área con mayores problemas y corregirlos en primeras instancias. 
Segundo Noé Quizhpilema-Acero; Ana Gabriela Hinojosa-Caballero; Renán Teodoro Rodríguez-Pillaga; Juan Edmundo Álvarez-Gavilanes

\section{FINANCIAMIENTO}

No monetario

\section{AGRADECIMIENTO}

A los emprendedores del cantón Cañar; por el apoyo en el desarrollo de la investigación.

\section{REFERENCIAS CONSULTADAS}

Aldana-Zavala, J. J. (2019). La competencia epistemológica en el investigador social universitario venezolano. [The epistemological competence in the Venezuelan university social researcher]. Praxis, 15(1), 103-115. https://doi.org/10.21676/23897856.3091

Cañar. (2014). Cañar (datos generales) [Cañar, general information]. Obtenido de Cañar: https://n9.cl/i9bk

El telégrafo. (28 de 06 de 2015). El cultivo de frutilla beneficia a 15 familias del cantón Cañar. [Strawberry cultivation benefits 15 families from the Cañar canton]. Recuperado de https://n9.cl/0wvs

Espinosa, R. (29 de 07 de 2013). La matriz de análisis dafo o foda. [The swot or swot analysis matrix]. Recuperado de https://n9.cl/vokx

Fundación Acción contra el Hambre. (2017). Diagnóstico sobre el emprendimiento social en Navarra ¿Qué necesidades y oportunidades existen para la implantación de una incubadora de emprendimiento social en Navarra? [Diagnosis of social entrepreneurship in Navarra What needs and opportunities exist for the implementation of a social entrepreneurship incubator in Navarra?]. Recuperado de https://n9.cl/dma2

INEC. (2010). Población y demografía. [Population and demographics]. Recuperado de https://n9.cl/9satz

Lasio, V, Ordeñana, X, Caicedo, G, Samaniego, A, Izquierdo, D. (2018). Global Entrepreneurship Monitor Ecuador 2017. Recuperado de https://n9.cl/7rf7

López, M. J. (2015). Estudio y aplicación de las actividades científico-tecnológicas. [Study and application of scientific-technological activities]. Madrid: UNED. Recuperado de https://n9.cl/tanh 
Segundo Noé Quizhpilema-Acero; Ana Gabriela Hinojosa-Caballero; Renán Teodoro Rodríguez-Pillaga; Juan Edmundo Álvarez-Gavilanes

Maqueda, J., \& Llaguno, J. I. (1995). Marketing estrategico para empresas de servicios. [Strategic marketing for service companies]. Madrid: Días de Santos, S.A. Recuperado de https://n9.cl/t69t9

Montilla, O. d. (2004). Modelo para evaluación de gestión de empresas industriales del subsector de cosméticos. [Model for management evaluation of industrial companies in the cosmetics subsector]. Recuperado de: https://n9.cl/9luj. scielo, 27.

Montilla-Galvis, O. (2004). Modelo para evaluación de gestión de empresas industriales del subsector de cosméticos. [Model for the evaluation of the management of industrial companies in the cosmetics subsector]. Estudios Gerenciales, 20(92), $25-45$.

Nuñis, L. (2017). Check-List para el diagnóstico empresarial. [Check-List for business diagnosis]. Barcelona: Profit Editorial I., S.L. Rescuperado de https://n9.cl/sw5I

Padilla, D. A., Erazo, J. C., \& Narváez, C. I. (2019). Diagnóstico situacional del potencial turístico de la parroquia Ricaurte, Azuay [Situational diagnosis of the tourist potential of the Ricaurte parish, Azuay] Revista Arbitrada Interdisciplinaria Koinonía, 380-381.

Padilla-Campoverde, D., Erazo-Álvarez, J., \& Narváez-Zurita, C. (2019). Diagnóstico situacional del potencial turístico de la parroquia Ricaurte, Azuay. [Situational diagnosis of the tourism potential of the Ricaurte parish, Azuay]. Revista Arbitrada Interdisciplinaria Koinonía, 4(1), 376-407. http://dx.doi.org/10.35381/r.k.v4i1.462

Reyes, P. E., Narváez, C. I., Erazo, J. C., \& Giler, L. V. (2020). Configuración del impuesto a la patente municipal con base al ingreso de las actividades económicas. Caso: GAD Municipal de Pucará - Ecuador. [Configuration of the municipal patent tax based on income from economic activities. Case: GAD Municipal de Pucará Ecuador]. Revista Espacios, 41(21), 197-211. Recuperado de https://n9.cl/8fvf

Riveros, N. (2010). Un diagnóstico de emprendimiento. [A diagnosis of entrepreneurship]. Recuperado de https://n9.cl/0qks

Rodas, D. N., \& Tituana, C. M. (2012). Estudio de pre-factibilidad para la producción y comercialización de la fresa en la ciudad de Cuenca. [Pre-feasibility study for the production and marketing of strawberry in the city of Cuenca]. Recuperado de https://n9.cl/pm6b 
Revista Arbitrada Interdisciplinaria KOINONIA

Año 2020. Vol V. №3. Especial: Administración

Hecho el depósito de Ley: FA2016000010

ISSN: 2542-3088

FUNDACIÓN KOINONIA (F.K). Santa Ana de Coro. Venezuela.

Segundo Noé Quizhpilema-Acero; Ana Gabriela Hinojosa-Caballero; Renán Teodoro Rodríguez-Pillaga; Juan Edmundo Álvarez-Gavilanes

Rodríguez-Piña, R, \& Peña-Domínguez, Z. (2008). Diagnóstico cultural del ambiente organizacional: un paso previo a la introducción de un sistema de gestión de información. [Cultural diagnosis of the organizational environment: a step prior to the introduction of an information management system]. ACIMED, 17(5).

Vidal, K., Erazo, J., \& Narváez, C. (2019). La lógica difusa como herramienta de evaluación financiera de proyectos de inversión. [Fuzzy logic as a tool for financial evaluation of investment projects]. Revista Arbitrada Interdisciplinaria Koinonía, 1(4), 309-348. http://dx.doi.org/10.35381/r.k.v4i1.460

(C2020 por los autores. Este artículo es de acceso abierto y distribuido según los términos y condiciones de la licencia Creative Commons Atribución-NoComercial-Compartirlgual 4.0 Internacional (CC BY-NC-SA 4.0)

(https://creativecommons.org/licenses/by-nc-sa/4.0/). 